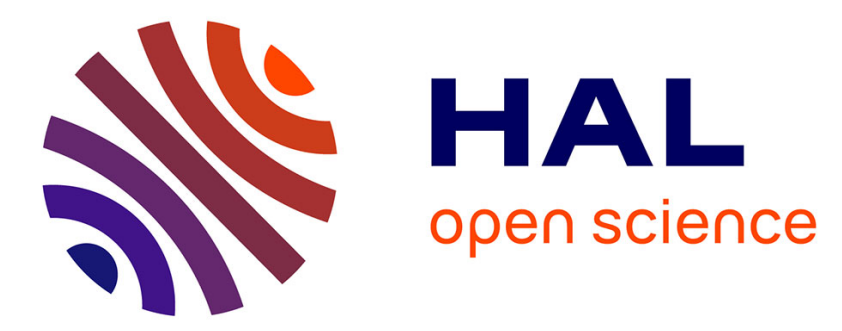

\title{
Les échographistes face au dépistage prénatal de la trisomie 21. Le difficile arbitrage entre excellence professionnelle et éthique du consentement Bénédicte Champenois-Rousseau, Carine Vassy
}

\section{- To cite this version:}

Bénédicte Champenois-Rousseau, Carine Vassy. Les échographistes face au dépistage prénatal de la trisomie 21. Le difficile arbitrage entre excellence professionnelle et éthique du consentement. Sciences Sociales et Santé, 2012, 10 (4), pp.39 - 63. 10.3917/sss.304.0039 . hal-01493095

\section{HAL Id: hal-01493095 \\ https://hal.science/hal-01493095}

Submitted on 20 Mar 2017

HAL is a multi-disciplinary open access archive for the deposit and dissemination of scientific research documents, whether they are published or not. The documents may come from teaching and research institutions in France or abroad, or from public or private research centers.
L'archive ouverte pluridisciplinaire HAL, est destinée au dépôt et à la diffusion de documents scientifiques de niveau recherche, publiés ou non, émanant des établissements d'enseignement et de recherche français ou étrangers, des laboratoires publics ou privés. 


\title{
Les échographistes face au dépistage prénatal de la trisomie 21. Le difficile arbitrage entre excellence professionnelle et éthique du consentement
}

\author{
Bénédicte Champenois-Rousseau*, Carine Vassy ${ }^{* *}$
}

\begin{abstract}
Résumé : Le suivi médical des femmes enceintes en France comporte aujourd'hui un dépistage de la trisomie 21 fotale, qui peut déboucher sur une interruption médicale de grossesse. L'une des principales techniques utilisées est l'échographie. Des autorités publiques ont fait des recommandations aux professionnels pour qu'ils demandent à la femme si elle consent au test avant de le réaliser, mais des critiques disent que le dépistage est devenu de fait quasi obligatoire. Notre recherche sociologique dans un cabinet de la région parisienne permet d'étudier le travail d'information effectué par les échographistes et de montrer que ceux-ci se satisfont souvent d'un consentement implicite de la femme enceinte.
\end{abstract}

Mots-clés : échographie, dépistage, consentement, trisomie, éthique,

La volonté de faire de la prévention est ancienne en médecine, mais depuis quelques décennies elle s'est amplifiée, portée par des innovations techniques inédites, qui permettent de détecter des risques de développer des maladies. C'est cette étape supplémentaire de la médicalisation, qu'A. Clarke a désignée sous le terme de biomédicalisation (Clarke, 2000). Celle-ci repose, entre autres, sur l'évaluation des risques de santé que court chaque individu, de manière à ce qu'il puisse ensuite prendre des mesures pour mieux les gérer. Ce changement implique une multiplication des dépistages possibles, tout au long de l'existence, et entraîne une modification du contenu des échanges entre médecins et profanes dans la consultation. Il ne s'agit plus seulement d'interpréter les symptômes d'un corps souffrant mais de raisonner en termes d'incertitude et de risque, à la fois pour estimer l'intérêt du dépistage et lors de la discussion des résultats de ce dernier, pour décider de la conduite à adopter. L'exercice est difficile car il nécessite d'appliquer des risques statistiques calculés sur des cohortes de patients, à l'individualité concrète d'une personne (Ménoret, 2007). Les pouvoirs publics ont pris des mesures pour améliorer l'information des personnes et le recueil de leur consentement. Suite à plusieurs textes de lois et actes de jurisprudence, la loi du 4 mars 2002 en France a consacré le droit des patients à être informés sur les investigations, les traitements et les actions de prévention qui leur sont proposés, faisant de l'information «un droit pour le patient et un devoir pour le professionnel » (Seguy, 2006).

Le dépistage prénatal des anomalies fœtales offre un exemple remarquable des problèmes liés à la biomédicalisation. Il permet d'obtenir des informations partielles sur un être en

\footnotetext{
*Bénédicte Champenois-Rousseau, sociologue, Institut de recherche interdisciplinaire sur les enjeux sociaux. (IRIS) Université Paris 13, UFR SMBH, 74 rue Marcel Cachin 93017 Bobigny cedex

** Carine Vassy, sociologue, Institut de recherche interdisciplinaire sur les enjeux sociaux (IRIS) Université Paris 13, UFR SMBH, 74 rue Marcel Cachin 93017 Bobigny cedex
} 
devenir, à partir desquelles il faut prendre des décisions difficiles. Dans un certain nombre de cas, les anomalies détectées ne sont pas curables et la solution proposée est l'interruption médicale de grossesse. La technique reine pour la détection des anomalies fœtales est l'échographie, qui consiste à interpréter des images obtenues à l'aide d'ultrasons envoyés pour explorer les tissus maternels et fœtaux. Cette technique s'est largement diffusée en France dans les années 1980 grâce à des obstétriciens et des radiologues (Fellous, 1988). Elle permet d'apporter aux praticiens et aux futurs parents un grand nombre d'informations : compter le nombre de fœetus, voir s'ils sont vivants, mesurer leur taille et donc déterminer le terme de la grossesse etc. L'usage de cette technique s'est aussi inscrit dans la politique de diagnostic prénatal d'une anomalie chromosomique, la trisomie 21, qui s'est développée à partir des années 70 en France (Vassy, 2011). Des échographistes ont montré que certaines caractéristiques du fœtus, comme des fémurs de petite taille, pouvaient être corrélées avec un risque accru de trisomie 21. Dans les années 1990, on a mis au point une mesure plus précise, celle de la nuque du fotus. Si celle-ci est plus épaisse que la moyenne entre 11 semaines et 13 semaines et 6 jours d'aménorrhée ${ }^{1}$, le risque de trisomie 21 est augmenté. Un logiciel peut en donner une estimation chiffrée à partir de la mesure de l'image (ou clarté) de la nuque et de l'âge de la femme, en l'exprimant sous forme de ratio (par exemple un risque de un sur cent) ${ }^{2}$.

Le dépistage de la trisomie 21 par échographie s'est développé en parallèle d'une autre technique, celle du dosage des marqueurs sériques de la trisomie 21 au second trimestre de la grossesse, qui repose sur l'analyse biochimique du sang maternel entre la 14e semaine et 17e semaine et 6 jours d'aménorrhée. Dans les deux cas, quand les valeurs mesurées à l'échographie ou par le dosage biochimique s'éloignent de la moyenne, le praticien doit proposer à la femme de faire un examen diagnostique pour déterminer si le foetus est réellement atteint. Il faut pour cela prélever du liquide amniotique par amniocentèse, ou du tissu placentaire, pour faire l'analyse génétique des cellules fotales (caryotype). Cet examen, qui s'accompagne d'un risque de fausse-couche généralement estimé à $1 \%$, permet de voir si le fœtus a une trisomie, mais ne prédit pas le degré de gravité de l'atteinte, par exemple en terme de sévérité de la déficience mentale. Néanmoins, quand le diagnostic est avéré, l'immense majorité des femmes enceintes choisissent d'avorter, comme le montre une étude en région parisienne (De Vigan et al., 2008).

Cette situation est critiquée depuis au moins deux décennies par des observateurs divers qui la qualifient d'eugéniste. Il s'agit de chercheurs en sciences sociales (Pinell, 2004 ; Moyse, 1999), d'hommes politiques (Mattei, 2000), de médecins (Milliez, 1999 ; Leblanc 2010), de porte-parole de l'Église catholique et de la fondation Lejeune. Ces observateurs jugent que ce dépistage prénatal aboutit à la disparition d'une catégorie de personnes jugées indésirables. Certains disent que ce dépistage est devenu systématique, et que les femmes enceintes n'ont pas toujours le choix de passer ou non les tests. D. Sicard (2007), ancien président du Comité consultatif national d'éthique (CCNE), va jusqu'à affirmer que le dépistage prénatal de la trisomie 21 est «devenu quasi-obligatoire ». Certains lient ce phénomène à une forme de judiciarisation de la médecine, qui fait craindre aux médecins que des parents d'un enfant né handicapé leur fassent un procès si tous les moyens de dépistage n'ont pas été mis en œuvre pendant la grossesse.

Pour contrer ces accusations d'eugénisme, crime d'ailleurs sanctionné par la loi, des instances éthiques ont émis des recommandations et les pouvoirs publics ont pris des mesures. Il s'agit de s'assurer que tous les tests de dépistage et de diagnostic de la trisomie 21 sont effectués avec l'accord de la femme enceinte, après que le praticien l'a informée de ces possibilités. La norme éthique ainsi affirmée est celle de l'autonomie du patient, qui

\footnotetext{
${ }^{1}$ L'aménorrhée désigne l'absence de règles.

${ }^{2} \mathrm{Ce}$ logiciel est élaboré à partir de données statistiques sur les mesures de nuque fœtale de fœtus trisomiques et non trisomiques.
} 
s'exprime à travers la notion de consentement éclairé. Ce concept a une longue histoire dans les textes juridiques internationaux et nationaux, ainsi que dans le système de santé français (Marzano, 2006). Des sociologues ont montré l'accroissement de l'exigence de consentement éclairé formalisé par écrit, tout en soulignant ses ambiguïtés dans le domaine de la procréation médicalement assistée et des soins palliatifs, ainsi que dans la recherche biomédicale (Ducournau, 2010; Memmi 2003). En ce qui concerne le consentement aux soins, l'anthropologue S. Fainzang (2006) a forgé l'expression de consentement résigné pour décrire l'attitude de certaines personnes gravement malades face à leur médecin.

L'encadrement éthique et juridique du dépistage de la trisomie 21 porte sur les marqueurs sériques et, dans une moindre mesure, sur l'échographie. Le CCNE a émis un avis sur le dépistage par les marqueurs en 1993, précisant que ce programme ne pourrait être mis en place que si la liberté de décision de la femme était respectée. En 1997, les pouvoirs publics ont décidé de faire financer ce test par l'Assurance-maladie, en précisant que ce test ne pourrait être accompli que si la femme a signé un formulaire de consentement. Pour le dépistage par échographie en revanche, aucun texte réglementaire n'oblige les praticiens à demander le consentement de la femme, mais des recommandations générales ont été émises par l'Agence nationale d'accréditation et d'évaluation en santé en 1998 : il faut pratiquer des échographies de grossesse "sous réserve de l'obtention du consentement éclairé de la femme enceinte » car ces examens peuvent déboucher sur le dépistage d'anomalies (ANAES, 1998, p. 54). Dans la décennie suivante, cette agence, devenue la Haute autorité de santé, émet des recommandations en matière d'information des femmes enceintes pour les deux types de dépistage de la trisomie 21. Les tests doivent être systématiquement proposés par les professionnels, mais sans être obligatoires. En ce qui concerne l'examen par échographie, la femme enceinte doit être informée que ce test a pour objectif, parmi d'autres, de dépister des anomalies fœtales (HAS, 2005, p.27). Dans un autre rapport en 2007, portant sur les stratégies de dépistage de la trisomie 21, elle rappelle que ces tests ne peuvent être accomplis qu'avec l'accord de la femme enceinte (HAS, 2007). La dernière partie du rapport, qui porte sur la «mise en cuvre des principes éthiques», formule des recommandations encore plus ambitieuses. Le praticien doit donner « une information simple, intelligible et adaptée sur la nature de l'examen, la signification des résultats, l'existence éventuelle d'une prévention ainsi que ses contraintes, les implications d'un recours ou d'un non-recours au dépistage, mais également sur la trisomie 21 elle-même, l'espérance de vie des personnes atteintes et les possibilités de développement et d'intégration sociale $($ HAS, 2007, p. 142).

L'affirmation du principe selon lequel les tests de dépistage ne doivent être accomplis qu'avec l'accord de la femme enceinte, se trouve cependant en contradiction avec des recommandations plus anciennes, qui émanent d'une instance différente : le Comité national technique de l'échographie de dépistage prénatal (CNTE), institué en 2001 par le ministre de la santé. Ce comité rassemblait les représentants des principaux syndicats et organisations professionnelles d'échographistes et d'obstétriciens, dans le but d'écrire un document de référence sur l'échographie fœtale, à destination des professionnels et du public, dans un contexte marqué par plusieurs affaires judiciaires. Son rapport remis en 2005 établit la liste des éléments que le praticien doit vérifier sur les fœtus à chaque échographie de dépistage et qui doivent figurer dans le compte-rendu d'examen, pour être transmis au médecin prescripteur et demeurer dans le dossier médical de la femme pendant toute la grossesse (Sureau et Henrion, 2005). En ce qui concerne l'échographie du premier trimestre, cette liste comprend la mesure de la nuque fœtale, tout en précisant : "après information spécifique, et si la patiente le souhaite, il peut être procédé à un calcul de risque d'anomalie chromosomique » (p.21). Il est donc demandé au praticien de mesurer la nuque, ce qui lui donne immédiatement des informations car il est capable d'interpréter ce chiffre brut, puis de parler à la femme enceinte du dépistage de la trisomie 21 et, si elle est intéressée, de lui 
communiquer le résultat calculé par le logiciel en fonction de la mesure et de son âge, pour estimer le risque.

Que font les échographistes dans ce contexte ambigu où il y a contradiction entre des normes instituées par une agence sanitaire au nom de l'éthique et d'autres normes professionnelles qui définissent l'excellence technique ? Comment se noue l'accord autour de la mesure, ou de l'absence de mesure, de la nuque fotale lors de leur interaction avec les femmes enceintes?

Quelques enquêtes ont été effectuées auprès de femmes ayant eu une grossesse en France pour connaître leur perception du dépistage échographique. Un quart des femmes ayant répondu au questionnaire de V. Seror et Y. Ville (2009) dans une maternité de la région parisienne disent n'avoir pas été informées que l'examen échographique du premier trimestre comportait une évaluation du risque de trisomie 21 et moins de la moitié des enquêtées avait conscience que les résultats de cet examen pouvaient entrainer une décision concernant la poursuite de leur grossesse. De même B. Khosnood (2006) a montré que des femmes ayant accouché dans un hôpital parisien avaient un faible niveau de connaissances sur la mesure de la nuque fotale, en particulier dans les catégories socioéconomiques défavorisées. Le constat de l'ignorance de certaines femmes amène à se demander si leur consentement a réellement été obtenu. La recherche de R. Favre (2007) apporte des éléments supplémentaires car elle s'intéresse aux femmes enceintes mais aussi à leurs médecins, pour apprécier la qualité des informations médicales données et la liberté de choix qui est laissée en matière de dépistage. Il a interrogé par questionnaire des médecins qui ont prescrit des échographies et leurs patientes dans l'Est de la France. Près des deux tiers des professionnels interrogés ont une mauvaise connaissance de l'échographie fotale, et l'immense majorité d'entre eux se disent personnellement favorables à ce dépistage. Il en déduit que la majorité des praticiens ont une position qui rend la recherche du consentement de la femme difficile, voire qui le néglige. Son enquête auprès des femmes enceintes montre que la grande majorité d'entre elles ont une attitude favorable au dépistage échographique, et que leur niveau de connaissances est fortement corrélé à celui de leur médecin. D'après lui, seul un quart d'entre elles ont exercé un consentement éclairé à ce dépistage.

Ces travaux corroborent les résultats de recherches effectuées en Angleterre et aux EtatsUnis sur la fragilité de la notion de consentement à propos des dépistages biologiques ou échographiques de la trisomie 21. Les femmes enceintes perçoivent les tests de dépistage de la trisomie 21 comme faisant partie de la routine du suivi médical des grossesses et n'ont pas forcément conscience qu'on leur demande d'effectuer un choix (Marteau et al., 1992 ; Press et Browner, 1997). Une fois le test passé, certaines femmes enceintes se trouvent en possession d'une information qu'elles n'ont pas anticipée, celle d'un risque élevé de trisomie 21 fœtale, qui les amène à envisager avec inquiétude, voire angoisse, la perspective de l'amniocentèse et de l'interruption médicale de grossesse (Katz-Rothman 1989, Rapp, 2000 ; Heyman et al., 2006). Du côté des praticiens, les professionnels interrogés par C. Williams (2002) reconnaissent avoir du mal à appliquer le principe de non-directivité prôné dans les guides de bonnes pratiques de dépistage du système de santé britannique et disent effectuer parfois des choix à la place de leur patiente «dans leur intérêt ».

Les recherches menées sur le dépistage prénatal par échographie, en France comme en Angleterre et aux Etats-Unis, sont utiles pour comprendre la perception qu'en ont les acteurs. Mais elles reposent souvent sur des choix méthodologiques, comme des questionnaires ou des interviews de praticiens et de femmes enceintes après les examens, qui ne permettent d'étudier les interactions qu'à travers les souvenirs qu'en ont les acteurs et le discours qu'ils construisent a posteriori. On ne sait donc pas comment les interactions se sont déroulées précisément, ni ce qui donne à certaines femmes enceintes le sentiment qu'on ne leur a pas 
laissé le choix, et à certains praticiens l'impression qu'ils ne peuvent pas faire autrement que d'être directifs.

Pour comprendre les pratiques des acteurs, une approche interactionniste fondée sur l'observation permet d'analyser ce que les acteurs font, car cela peut être différent de ce qu'ils disent faire (Strauss, 1992 ; Péretz, 1998). A notre connaissance, seule la sociologue anglaise A. Pilnick (2008) a analysé systématiquement les paroles échangées dans des consultations à propos du dépistage prénatal par échographie. Elle a été autorisée à enregistrer des consultations de sages-femmes, sans pouvoir les observer, où les professionnelles présentent aux femmes enceintes le dépistage échographique de la trisomie 21. Les sages-femmes n'attendent pas un accord ou un refus immédiat pour le test, mais elles délivrent des informations afin que les femmes enceintes donnent ou non leur consentement au dépistage quand elles iront faire leur échographie à l'hôpital. Dans cette étude, toutes les femmes ont accepté le calcul de risque échographique. A. Pilnick souligne deux facteurs explicatifs. Tout d'abord le choix du moment de l'évocation du test par la sage-femme joue un rôle essentiel. Le test est généralement présenté après les tests sanguins qui sont recommandés pour la santé de la femme et du fœtus car ils permettent de dépister des maladies pour lesquelles il existe des traitements (maladies sexuellement transmissibles, anémies etc.). Cela accentue l'impression que le dépistage échographique fait partie des examens fortement conseillés. De plus le dépistage de la trisomie 21 est toujours décrit en termes laudatifs : "c'est le meilleur test qu'on ait en ce moment », même si les sages-femmes précisent toujours qu'il n'est pas obligatoire.

Les résultats de cette étude ne sont pas transposables au contexte français car le suivi des femmes enceintes et la répartition du travail d'information parmi les professionnels n'y sont pas organisés de la même manière. En France, les soignants qui reçoivent une femme enceinte venue déclarer sa grossesse pour la première fois sont principalement des médecins généralistes ou gynécologues libéraux. Ils lui prescrivent plusieurs tests, dont l'échographie, et ne lui donnent pas nécessairement une information détaillée sur le dépistage de la trisomie 21 par mesure de la nuque car ils pensent que c'est du ressort de l'échographiste. Dans l'enquête de Favre (2007), près de 7\% des médecins disent ne donner aucune explication préalable à l'échographie. Il n'y a pas de répartition claire du travail d'information entre le médecin et l'échographiste, qui ne se connaissent d'ailleurs pas nécessairement, car la femme est libre de prendre rendez-vous pour l'examen parmi les échographistes libéraux disponibles à proximité. Le travail d'information sur le dépistage peut donc être effectué uniquement pendant l'examen échographique, même si cela ne représente vraisemblablement pas la majorité des cas.

\section{Méthodologie et échantillon}

Pour étudier comment se déroulent les interactions autour du dépistage de la trisomie 21 fœtale, nous avons choisi d'analyser 28 consultations. Celles-ci ont été observées et enregistrées par Bénédicte Champenois-Rousseau dans un centre d'échographie d'avril à juillet 2008. Le centre est un cabinet libéral en région parisienne, spécialisé dans l'échographie gynécologique et obstétrique. Nous l'avons choisi car les associés sont des professionnels très actifs au sein d'une association professionnelle de spécialistes de l'échographie fotale, reconnus par leurs pairs comme de bons professionnels, et qui travaillent régulièrement avec des médecins hospitaliers, qui leur envoient des patientes pour avoir un second avis. Ils ont mis en œuvre des outils pour informer les femmes enceintes sur un site Internet et dans la salle d'attente du cabinet. Par exemple, la secrétaire donne aux femmes qui viennent pour une échographie du premier trimestre une fiche décrivant les 
objectifs de l'examen, et notamment les tests de dépistage prénatal de la trisomie 21 . L'ensemble des 8 opérateurs que nous avons sollicités lors d'une réunion de présentation de la recherche à leur cabinet, soit 6 médecins (dont 4 femmes) et 2 sages-femmes, a accepté notre présence. Ils étaient avertis que notre enquête portait sur la communication autour du dépistage de la trisomie 21 et ont peut-être modifié leur comportement en conséquence pendant les observations ${ }^{3}$. En ce qui concerne les femmes enceintes, l'observatrice, qui était habillée en civil et ne portait pas de blouse blanche, leur demandait dans la salle d'attente, avant leur consultation, si elles accepteraient d'être observées et enregistrées dans le cadre d'une recherche sur la communication autour du dépistage de la trisomie 21. En cas de réponse positive, la sociologue rentrait alors dans la salle d'échographie avec la femme. Un petit nombre de femmes a refusé la présence de l'observatrice. Après l'enquête, nous n'avons conservé dans l'échantillon que les consultations de femmes enceintes de 11 à 13 semaines et 6 jours d'aménorrhée. Ce n'est que dans cette période que la mesure de la nuque est interprétable par le logiciel de calcul du risque de trisomie 21 fotale. Nous avons aussi exclu de l'échantillon les consultations de femmes qui avaient déjà eu une échographie pour cette grossesse avant la $11^{\mathrm{e}}$ semaine, car elles étaient susceptibles d'avoir eu des informations sur le dépistage de la trisomie 21 fœtale lors de l'échographie précédente et donc de ne plus bénéficier de l'information habituelle le jour de l'observation. Nous avons aussi exclu quelques cas de femmes à qui l'échographiste annonçait un risque de trisomie 21 fœtale élevé au cours de l'examen car celui-ci changeait de discours rapidement pour aborder le thème du test diagnostique et la question du consentement au prélèvement. Ces consultations ont été ensuite entièrement retranscrites pour pouvoir analyser les termes dans lesquels l'échographiste présente le dépistage, les prises de parole de la femme enceinte, ainsi que le moment et la durée de l'évocation de ce sujet dans la consultation. Les retranscriptions ont été complétées par les notes d'observation pour pouvoir inclure l'étude des interactions non verbales.

\section{Un dispositif sociotechnique contraignant la communication}

Pour analyser la communication entre les acteurs, il faut prendre en compte les contraintes liées au dispositif sociotechnique qu'est la consultation d'échographie. Afin de réussir à produire des images échographiques utiles pour le suivi de grossesse, un agencement de personnes et d'objets doit coopérer à un moment précis de leur trajectoire. Pour rentabiliser les onéreux échographes qui équipent le cabinet, tout en satisfaisant aux critères professionnels de qualité, la durée des consultations est fixée à 30 minutes. Dans ce délai, l'échographiste travaille à produire des images de différentes parties du foetus, qui répondent à des critères établis dans la littérature internationale (type de coupe, grossissement, netteté etc.). Faisant des mesures au millimètre, voire au dixième de millimètre pour la nuque, sur un fotus qui ne mesure que quelques centimètres, il a besoin de se concentrer et reste souvent les yeux rivés sur l'écran pendant l'examen. Certains praticiens parlent peu pendant cette partie de la consultation. Si les images produites sont mauvaises, leur réputation professionnelle auprès des autres professionnels qui suivront la grossesse sera en jeu, dans un milieu où la qualité des échographies est variable.

\footnotetext{
${ }^{3}$ Mais nous ne pensons pas que ce phénomène se soit produit, car la sociologue était assez expérimentée pour faire oublier sa présence pendant une enquête relativement longue, faisant suite à des observations similaires faites pour sa thèse (Champenois-Rousseau, 2003). Par exemple pendant les consultations, elle était assise en dehors du champ de vision de l'échographiste. De plus les résultats d'enquête ne montrent pas d'influence indue de l'observatrice.
} 
Des contraintes temporelles pèsent aussi sur la femme. Pour que l'on puisse évaluer le risque de trisomie 21 fœtale, elle doit se présenter à un moment précis de la grossesse, entre 11 et 13 semaines plus 6 jours d'aménorrhée, ce qu'a vérifié la secrétaire avec qui elle a pris rendez-vous par téléphone. Mais cette estimation s'avère parfois fausse, rendant impossible le calcul de risque de la trisomie 21 pendant la consultation. La femme subit également des contraintes corporelles qui peuvent avoir un retentissement émotionnel : pour pouvoir passer la sonde à ultrasons sur le ventre, l'opérateur lui demande de baisser ses vêtements sur ses hanches, ou de se déshabiller en dessous de la ceinture, avant de s'allonger sur un lit d'examen. Cet écart par rapport aux normes habituelles de la pudeur met certaines femmes mal à l'aise et peut gêner leur prise de parole. Parfois l'atteinte à la pudeur est encore plus forte. En effet, divers obstacles peuvent empêcher la mesure de la nuque : le fœetus n'est pas dans la bonne position, le cordon ombilical peut passer devant son cou, la femme peut avoir une surcharge pondérale au niveau de l'abdomen qui gêne le passage des ultrasons etc. L'opérateur peut essayer de changer la position du fotus en tapotant ou poussant sur le ventre, ou proposer à la femme de revenir dans quelques heures ou jours, pour que le fœtus change de position, ou encore choisir d'introduire la sonde dans le vagin de la femme, pour voir le fotus sous un autre angle. Il met alors sa main entre les cuisses de la femme. Cette contrainte est similaire à celles des consultations gynécologiques, où l'examen a une dimension intime, voire potentiellement sexuelle, qui doit être neutralisée par la patiente et le praticien, même quand ce dernier est de genre féminin (Guyard, 2002). Quand il n'y a pas d'obstacles à la mesure de la nuque, certains échographistes choisissent quand même de faire des clichés intra vaginaux pour augmenter le nombre d'images et diminuer ainsi le risque d'erreur de mesure. Plus de la moitié des consultations de notre échantillon comportent ce type de clichés.

Dans tous les cas, la réussite de la mesure suppose un travail d'alignement de la sonde et des corps du fœtus, de la femme, et de l'opérateur, qu'il faut obtenir à un moment précis de la grossesse et sans y passer plus de quelques dizaines de minutes. A la concentration intellectuelle de l'opérateur répond le travail corporel et émotionnel de la femme enceinte, qui doit s'autocontrôler dans une position physique qu'elle peut percevoir comme indécente et face aux enjeux psychologiques de sa grossesse mise en image. Ces contraintes pèsent sur la communication. Les moments qui précèdent et qui suivent l'examen sont peut-être plus favorables. Dans ce que nous avons appelé l'introduction de la consultation, l'échographiste fait entrer la femme dans la salle, puis ils discutent assis de part et d'autre d'un bureau ${ }^{4}$. L'échographiste pose quelques questions à la femme sur sa grossesse et ses antécédents médicaux. Dans la conclusion, la femme se rhabille et ils reviennent s'asseoir autour du bureau. L'opérateur fait part de ses remarques finales, rédige un compte-rendu sur l'ordinateur, l'imprime et le donne à la femme enceinte, avant de lui signifier la fin de la consultation.

\section{Les temporalités de l'information}

Si la communication est marquée par des contraintes issues du dispositif sociotechnique, elle varie aussi en fonction des choix faits par les échographistes pour présenter l'examen, en particulier du moment choisi ${ }^{5}$. Dans 10 consultations, ils l'évoquent pour la première fois

\footnotetext{
${ }^{4}$ Dans trois consultations de notre échantillon, la salle est trop exiguë pour contenir un bureau, et la femme est invitée à s'asseoir sur le lit d'examen.

${ }^{5}$ Ce sont les échographistes qui abordent le sujet du dépistage de la trisomie 21 en premier, et non les femmes enceintes. D'une manière générale, ils conduisent les interactions verbales, en posant des questions, ou en faisant des commentaires, par exemple sur les images qu'ils produisent. Dans deux consultations, la femme prend l'initiative de parler du dépistage, mais l'opérateur clôt le sujet en lui disant qu'il va l'aborder plus tard.
} 
dans la partie introductive. Dans 16 consultations, ils le font pendant l'examen, et dans deux consultations lors de la conclusion. Chacun évoque toujours le dépistage au même moment, sauf une opératrice qui varie et que nous avons nommée $\mathrm{C}$. La majorité des opérateurs en parle donc au moment de l'examen, quand ils voient apparaître la nuque à l'écran.

Tableau : Moment de première évocation du dépistage de la trisomie 21 dans la consultation échographique

\begin{tabular}{|c|c|c|c|}
\hline $\begin{array}{c}\text { Moment de première } \\
\text { évocation du dépistage }\end{array}$ & $\begin{array}{c}\text { Nombre } \\
\text { de } \\
\text { consultations }\end{array}$ & Opérateurs & $\begin{array}{c}\text { Nombre } \\
\text { d'opérateurs } \\
\text { concernés }\end{array}$ \\
\hline Introduction & 10 & $\mathrm{~B}, \mathrm{C}, \mathrm{F}$ & 3 \\
\hline $\begin{array}{c}\text { Examen } \\
\text { échographique }\end{array}$ & 16 & $\mathrm{~A}, \mathrm{C}, \mathrm{D}, \mathrm{E}, \mathrm{G}$ & 5 \\
\hline Conclusion & 2 & $\mathrm{C}, \mathrm{I}$ & 2 \\
\hline Total & 28 & - & - \\
\hline
\end{tabular}

Nous allons montrer que les choix de temporalité faits par les opérateurs leur ouvrent ou ferment des possibilités, ainsi qu'aux femmes enceintes, et que cela facilite plus ou moins la prise en compte des souhaits exprimés par les femmes.

\section{Parler du dépistage avant l'examen : une dissociation purement formelle ?}

Evoquer le dépistage dans la partie introductive de la consultation est conforme aux recommandations de la Haute autorité de santé qui insiste sur la nécessité d' "informer clairement la femme de son droit d'accepter ou de refuser un examen de dépistage (qu'il soit obligatoire, ou non obligatoire mais proposé systématiquement), après lui avoir expliqué, avant sa réalisation, l'utilité, les bénéfices escomptés et les éventuels inconvénients et conséquences de cet examen » (HAS, 2005) (souligné par nous). L'agence présente ce qui est considéré comme la meilleure démarche du point de vue de l'éthique médicale et demande que le sujet du dépistage soit abordé avant que le cours de l'action soit engagé. Dans la consultation d'échographie, le choix de ce moment permet à la femme de s'exprimer quand elle est encore dans une position physique et psychologique proche de celle de la vie quotidienne. C'est un moment où sa participation active est sollicitée pour confirmer les informations figurant dans son dossier, et décrire ses grossesses précédentes, le cas échéant.

Sur les dix consultations, seules trois comportent une demande explicite de consentement au dépistage de la trisomie 21, avec un opérateur qui utilise une tournure interrogative pour demander à la femme si elle souhaite le faire. Par exemple : «Vous souhaitez le faire le dépistage ou pas? »[F1] $]^{6}$, ou «Et donc cette fois-ci, vous le faites? » [B1]. Dans les autres consultations, il n'y a pas de demande explicite. Le praticien aborde toujours le sujet en demandant à la femme si elle a lu la fiche d'information qui lui a été donnée pendant qu'elle patientait en salle d'attente, ce à quoi la femme répond positivement ou ne répond pas. Quand elle répond positivement, ceci est souvent interprété comme un consentement au dépistage, comme dans l'extrait suivant, où l'opératrice change immédiatement de sujet pour demander à la femme si sa famille souffre de problèmes héréditaires. Plus tard, l'opératrice annonce qu'elle fait la mesure.

Opératrice C. : ( $3^{\mathrm{e}}$ minute) Vous avez eu le temps de lire le papier bleu là ?

\footnotetext{
${ }^{6}$ La lettre entre crochets désigne le nom fictif de l'opérateur, et est suivie par le numéro de la consultation.
} 
Femme : Oui.

Opératrice C. : ... le grand papier bleu...

Femme : Oui.

Opératrice C. : ... Bon, rien de particulier dans la famille... (...)

Opératrice C. : ( $10^{\mathrm{e}}$ minute) Madame $G$. on va regarder par voie vaginale...

Femme : D'accord.

Opératrice C. : Hein, pour bien faire la mesure de nuque...

Femme : D'accord...[C1]

Pour cette échographiste, le fait d'avoir préparé et mis à disposition des femmes enceintes une fiche d'information à lire dans la salle d'attente suffit à les informer. Ceci ne correspond pas aux recommandations de l'ANAES (2000) sur l'information des patients, qui précise que l'information écrite ne peut être qu'un complément au dialogue oral. Dans quelques autres consultations, après la question sur la lecture de la fiche, l'opérateur demande à la femme si elle veut plus d'explications et si elle a déjà fait ce test lors d'une précédente grossesse. Une réponse positive à cette dernière question peut également être interprétée comme un consentement au dépistage pour la grossesse en cours :

Opérateur B. : ... OK! ...Vous avez lu le papier ...

Femme : Oui.

Opérateur B. : ... sur le dépistage de la trisomie 21 ?... C'était déjà comme ça pour votre premier bébé ?

Femme : Euuhhh... j'avais eu une prise de sang oui...

Opérateur B. ... Donc ... vous aviez eu l'échographie plus une... prise de sang ?

Femme : ... Prise de sang...

Opérateur B. : ... Il y a des choses que vous n'avez pas comprises, que vous vouliez éclaircir... il y a ...

Femme : Non.

Opérateur B. : ... Non ? D'accord.

Femme : Non, a priori... non...

Opérateur B. : ... Donc on va vous ... on va calculer un risque aujourd'hui à partir de... la mesure de la nuque qu'on va...

Femme : Hum...

Opérateur B. : ... faire... et de votre âge....

Femme : Ouais [B5]

Comme le montre l'utilisation du futur proche «on va calculer un risque aujourd'hui... », l'opérateur considère que si la femme a déjà fait le dépistage pour une précédente grossesse, et qu'elle ne demande pas plus d'explications, elle consent à le refaire. En revanche un précédent refus de dépistage pour une grossesse antérieure n'est pas considéré comme le signe d'un refus pour la grossesse en cours. C'est le cas d'une consultation où l'opératrice fait une demande explicite de consentement, mais où la femme est hésitante et signale son refus antérieur.

Opératrice F. : (...) Donc euh... vous avez lu le papier euh... qu'on vous a donné euh... Femme : Oui.

Opératrice F. : Euh d'accord. Est-ce que vous souhaitez le faire, est-ce que vous avez des questions à me poser? Vous souhaitez faire le dépistage ou pas?

Femme : Ben oui, je sais pas moi... (à son compagnon:) T'en penses quoi ?

Opératrice F. : Je sais pas, est-ce que vous avez des questions, est-ce que... 
Femme : En fait c'est notre deuxième, on l'avait pas fait pour le premier, donc euh... je sais pas... [F1]

L'opératrice donne ensuite des informations techniques sur la mesure de la nuque et le calcul de risque, puis demande si le médecin qui suit la grossesse a prescrit le dépistage biochimique. La femme répond qu'elle l'a refusé. Son compagnon renchérit en disant qu'ils avaient l'impression que le médecin leur « forçait la main ». L’opératrice réagit :

Opératrice F. : Bon, je vais vous expliquer moi, ce que je pense déjà... Moi je pense que je ne vous force pas, c'est à dire que moi ce que je veux, c'est que vous compreniez exactement ce que vous faites. Bon. Si vous me dites moi, de toute façon, je ne cherche pas l'information, si j'ai un enfant trisomique, je l'accepterai comme un autre, dans ce cas-là c'est pas la peine de faire le dépistage...

Femme : Ouais, c'est-à-dire on part un peu sur cette donnée là... C'est que pour nous, euh... On acceptera l'enfant euh... voilà...

Opératrice F. : Bon, maintenant, vous acceptez l'enfant, est-ce que vous voulez...

Femme : Après, s'il est hydrocéphale, je...

Opératrice F. : Non, non, mais ça n'a rien à voir...

[L'opératrice et la femme parlent de l'hydrocéphalie]

Opératrice F. : ... c'est-à-dire que vous dites bon, moi j'accepterai un enfant trisomique, est-ce que vous voulez savoir avant, ou est-ce que vous ne voulez pas de toute façon? Est-ce que vous préférez ne pas savoir?

Femme : Et si euh... Si euh... je dis non aujourd'hui, est-ce que je peux quand même le savoir à six mois par exemple?

Opératrice F. : Alors. Si vous dites non aujourd'hui, moi je mesure quand même la nuque, je vous le dis tout de suite, je vais mesurer la nuque quand même, je vais essayer de la mesurer le mieux possible quand même...

Femme : Hum.

Opératrice F. : Je vous donnerai pas... je la note et je ne vous donnerai pas la suite. [F1]

Devant les réticences de la femme, l'opératrice est amenée à expliciter sa position : si la femme n'est pas intéressée par le dépistage, l'échographiste va le faire quand même, mais sans lui communiquer le résultat. Le professionnel dit que son droit à produire cette connaissance outrepasse le droit de la femme à ne pas savoir. Dans la suite de la consultation, la femme accepte le dépistage et l'opératrice apporte un argument supplémentaire en disant que quand elle mesure une nuque très épaisse, elle communique cette information immédiatement à la femme enceinte, même si celle-ci ne le souhaite pas, car ce signe peut être associé à des anomalies cardiaques, dont certaines nécessitent des soins dès la naissance. Ce discours montre que l'échographiste est persuadée qu'il vaut mieux faire le dépistage pour le bien de l'enfant. L'ensemble de ces consultations montre que le choix d'évoquer le dépistage en début de consultation n'amène pas toujours le praticien à rechercher un consentement explicite, ni à respecter les hésitations d'une femme enceinte. Nous allons maintenant voir si l'évocation du dépistage pendant l'examen donne plus de possibilités de parvenir à des accords explicites.

\section{Informer sur le dépistage pendant qu'on l'effectue}


Quatre opérateurs ont évoqué pour la première fois le dépistage pendant l'examen échographique, au moment où ils obtiennent sur l'écran le profil sur lequel ils vont pouvoir faire les mesures. Cette temporalité ne paraît pas très propice à la discussion, du fait des contraintes que le dispositif sociotechnique fait peser sur la communication. Si certains opérateurs font des commentaires pour expliquer ce qu'on voit à l'écran, peu se lancent dans des explications approfondies et les femmes parlent peu également.

Comme dans les consultations où le sujet est abordé en introduction, on observe des accords implicites, basés sur des questions portant sur la lecture de la fiche d'information, ou sur le test de dépistage effectué lors d'une grossesse précédente. Une réponse positive à ces questions est interprétée comme un accord pour la mesure de la nuque.

Opératrice D. : Vous avez lu la petite feuille d'information sur le dépistage de la trisomie ou pas?

Femme : Oui.

Opératrice D: Donc la nuque elle est toute fine, donc ça correspond vraiment à un risque faible. [D3]

L'opératrice abandonne ensuite ce sujet jusqu'à la conclusion de l'examen. Elle n'a pas demandé à la femme si elle était d'accord pour faire le dépistage et elle lui a donné immédiatement une indication qualitative sur le résultat. Elle réalise un dépistage systématique, en limitant l'information au minimum, peut-être dans le but de ne pas inquiéter la femme enceinte. Dans d'autres consultations, le praticien ne cherche aucun accord sur le dépistage, même sur un sujet connexe comme la lecture de la fiche. Dans l'extrait suivant, l'opératrice présente le test comme une chose « importante » qui «doit » être faite.

Opératrice E : (...) Il (le bébé) tourne le dos hein! En fait j'ai besoin de l'avoir dans un plan de profil de la tête aux fesses pour deux choses importantes... à savoir dater la grossesse en le mesurant de la tête aux fesses et on doit faire la mesure de la clarté nucale pour le dépistage de la trisomie 21. On l'a eu un moment vers nous, là il est revenu à l'arrière. Alors là, il est sur un profil parfait sauf qu'il a le bout du nez qui vient se coller contre la paroi, donc on voit à peine son bout du nez ici (elle le désigne du doigt sur son écran), mais là il est exactement sur la coupe dont on a besoin pour faire les mesures. [E1]

L'opératrice décrit les plans de coupe qu'elle fait apparaître, et elle détaille les contraintes techniques qui pèsent sur son travail : par exemple si l'on ne voit pas le nez du fotus, la mesure ne sera pas considérée comme correcte. Elle ne présente pas les conséquences éventuelles du test, mais les conditions techniques de sa réussite. Ce faisant, elle garde la parole et la femme ne dit pas un mot. Dans la suite de son monologue, quand elle a pris les mesures, elle annonce le résultat en disant que «là en l'occurrence, (la nuque) c'est bien fin, donc le risque pour le bébé sera faible ». Elle ajoute immédiatement qu'elle ne fera le calcul du risque que si les parents le souhaitent. Les parents n'ont donc pas le droit de s'opposer au dépistage, ni d'ignorer la première information qualitative sur le résultat. Ils peuvent seulement refuser d'en connaître l'estimation chiffrée.

Cependant informer sur le dépistage au moment de l'effectuer n'empêche pas de demander explicitement le consentement de la femme. Dans l'extrait suivant, l'opératrice le demande à une femme accompagnée de son partenaire qui reste silencieux, et elle accepte le refus de la femme :

Opératrice C : Est-ce que vous faites le dépistage pour la trisomie 21 ? 
Femme : Non, on va pas le faire.

Opératrice C. : Vous l'aviez pas fait pour le précédent?

Femme : Non!

Opératrice C. : D’accord! [C5]

L'opératrice renonce à mesurer la nuque à l'écran. Quelques minutes plus tard, elle redemande sous forme interronégative si elle doit faire la mesure : «Donc je ne fais pas la mesure de la nuque?». La femme réitère son refus. Cette consultation est la seule de notre échantillon où la clarté nucale n'est pas mesurée du fait d'un accord entre l'opérateur et la femme enceinte. Les autres occurrences d'absence de mesure sont dues à des conditions d'examen défavorables : fœus mal positionné ou présence du cordon dans la zone à mesurer. Parler du dépistage au moment de l'examen peut donc fournir à l'échographiste l'occasion de demander explicitement à la femme son consentement au test, et à celle-ci d'exprimer clairement son refus. Le cours de l'action est encore réversible.

\section{N'évoquer le dépistage qu'après l'avoir fait}

Dans notre échantillon, seules deux opératrices dans deux consultations évoquent le dépistage en conclusion. Elles mesurent la nuque à l'écran sans rien dire et n'abordent le sujet que lorsque la femme s'est rhabillée et est revenue au bureau. Nous avions fait l'hypothèse initiale que lorsque le praticien adopte cette stratégie de communication, il donne moins de possibilité à la femme enceinte de faire un choix autonome car il détient déjà l'information sur le résultat quand il en parle. Cette hypothèse ne tient pas, comme le montre l'extrait suivant où l'opératrice vient de préciser à la femme l'âge du fœtus, puis enchaine sur le dépistage, tout en favorisant la libre expression de la volonté de la femme :

Opératrice I : Autrement, euh... donc... on a la possibilité de vous proposer un test de dépistage par rapport à la trisomie $21 . .$. donc vous avez lu le ... petit papier...

Femme : Oui.

Opératrice I : ... qu'on vous a donné... Oui! ... Donc euh c'est un test qu'on propose à toutes les... toutes les mamans...

Femme : D'accord.

Opératrice I : ... Donc euh... de tout âge... et avec toute... euh... tous résultats ... autrement... c'est donc pour vous rassurer de ce côté-là aussi donc ... euh... au même titre qu'on regarde euh... sur le plan morphologique si...

Femme : Humhum.

Opératrice I : ... tout est là.

Femme : Hum.

Opératrice I : Est-ce que vous souhaitez qu'on regarde euh... qu'on... que je fasse des calculs par rapport à ça?

Femme : C'est-à-dire, les calculs?

Opératrice I : ... Les calculs c'est que... on ... Hum !... on ...

Femme : Il n'y a pas de test physique à faire?

Opératrice I : Non ... il n'y a pas de test physique à faire...

Femme : Alors oui... [I 1]

L'opératrice insiste sur le caractère non obligatoire du test, en disant : «on propose » et «vous souhaitez?». Elle ne signale pas que la seule mesure de la nuque lui suffit à avoir déjà une idée de l'importance du risque de trisomie 21. Elle fait une présentation assez neutre du 
dépistage, sauf lors d'une occurrence franchement favorable au test, qu'elle décrit comme ce qui permet de «vous rassurer ». Les réactions de la femme montrent que la lecture de la fiche n'a pas suffi à sa compréhension car elle évoque un «test physique », ce qui est peut-être une allusion à l'amniocentèse. Après avoir obtenu des explications supplémentaires, la femme signifie clairement que cette information l'intéresse.

Cependant ce moment d'évocation du dépistage peut aussi permettre aux praticiens d'imposer leur connaissance du résultat du test. Dans l'extrait suivant, l'opératrice est face à une femme plutôt réticente au dépistage, qui mentionne qu'il n'est pas obligatoire puis reste laconique.

Opératrice $\mathrm{C}\left(22^{\mathrm{e}}\right.$ minute): Donc vous avez... vous faites la... le dépistage de la trisomie 21 ou pas?

Femme (inaudible, n'a pas l'air décidée)

Opératrice C : ... Qu'est-ce que vous avez compris dans... ce qui était noté... votre médecin vous en a parlé?

Femme : On peut le faire mais c'est pas obligatoire...

Opératrice $\mathrm{C}:$ Euh... le dépistage, non, rien n'est obligatoire... hein ? Le dépistage c'est pas... c'est... c'est juste un... un chiffre qu'on vous donne... par rapport à votre âge...

Femme : Hum.

Opératrice C : ... à la mesure de la nuque de votre bébé que j'ai mesurée aujourd'hui euh... et puis après une prise de sang... ça c'est le dépistage...

Femme (presque muette): Hum. [C 4]

Face à ces réticences, l'opératrice donne des informations techniques sur le déroulement du dépistage, à savoir la possibilité de combiner le résultat de l'échographie avec celui des marqueurs sériques, puis éventuellement de faire une amniocentèse et un caryotype. Elle demande à la femme si sa gynécologue lui a parlé du dépistage, et la réponse est négative. L'opératrice reprend :

Opératrice $\mathrm{C}:\left(23^{\mathrm{e}}\right.$ minute) C'est quelque chose qui vous inquiète... c'est quelque chose que vous voulez faire ou pas?

Femme : Ben je peux le faire quand même mais... bon... parce que ma tante elle m'a conseillé de le faire mais...

Opératrice C : Oui...

Femme : Je vois pas trop l'intérêt de...

Opératrice C : Oui.

Femme : Je sais pas moi...

Opératrice C: Pas trop l'intérêt pourquoi ? Parce que... euh... quoi qu'il arrive, vous gardez cet enfant... vous voulez pas savoir?

Femme : Ouais, voilà, quoi! De toute façon quoi qu'il arrive, je le garde... hein...

Opératrice C : D'accord... Alors ça c'est... c'est votre choix hein ? Vous êtes pas obligée du tout de faire un... dépistage euh... si pour vous euh... vous en voyez pas l'utilité hein? Hein?

Femme : D'accord.

Opératrice C: Humhum...

Femme : Je sais pas, de toute façon je vais parler avec le gynéco, c'est lui qui va me conseiller...

Opératrice C : Bon... Moi, en tout cas, j'ai quand même fait la mesure de nuque...

Femme : Hum. 
Opératrice C : Je vous dis quand même le chiffre... ou... vous voulez pas du tout...

Femme : Oui, allez-y... [C4]

Le discours de l'échographiste est ici paradoxal : elle demande explicitement à la femme enceinte son consentement et elle lui confirme à deux reprises que le dépistage n'est pas obligatoire, mais elle insiste tellement qu'elle finit par emporter son adhésion. Pour cela, elle dit deux fois qu'elle a déjà fait la mesure de la nuque, en cherchant à minimiser l'importance du test: «c'est juste un chiffre ». Et elle demande trois fois à la femme son consentement, jusqu'à ce que celle-ci accepte. Les arguments de la femme qui est opposée à la finalité abortive du dépistage et qui voudrait en parler avec son médecin traitant, ne sont pas pris en compte. Il est vrai que la femme a aussi montré à plusieurs reprises des hésitations : «je sais pas », et qu'elle n'a pas exclu complètement la possibilité d'être intéressée par cette information plus tard.

C'est la même opératrice C. qui n'a pas respecté les réticences de cette femme enceinte vis-à-vis du dépistage et qui a accepté le refus d'un couple de faire le dépistage au moment de l'examen dans la consultation C5 citée plus haut. On voit là l'importance de la capacité de négociation des acteurs et du moment où le sujet est évoqué. Dans la consultation où l'échographiste insiste pour donner le résultat du dépistage, la femme est seule et elle exprime beaucoup de réticences, plutôt qu'un refus net. Le fait que l'opératrice fasse la demande de consentement à la fin de la consultation a aussi une importance. Elle a déjà fait la mesure, donc elle est détentrice de l'information au moment où elle évoque le dépistage, et cette information est rassurante. Cela met vraisemblablement la praticienne dans une dynamique où il lui est difficile de s'abstenir de communiquer une bonne nouvelle. De plus une fois qu'elle est détentrice de l'information, et qu'elle a dit qu'elle l'était, il est peut-être plus difficile pour la femme enceinte de justifier un refus de connaître le résultat. En revanche, dans la consultation où $\mathrm{C}$. a accepté le refus du dépistage, la femme enceinte est accompagnée du futur père. Celui-ci ne dit mot, mais ne contredit pas son épouse quand elle répond immédiatement qu'elle n'est pas intéressée par le dépistage. Le discours de la femme enceinte est dénué d'ambiguité ou d'hésitation : c'est un refus net. De plus l'échographiste a évoqué le dépistage au moment de l'examen, juste avant de faire la mesure. Il lui est peut-être plus facile de s'abstenir de faire le test, quand le refus intervient tôt.

\section{Conclusion}

Les échographistes français sont placés devant des injonctions contradictoires en ce qui concerne le dépistage de la trisomie 21 fotale. Notre étude dans un cabinet de la région parisienne montre que le travail qu'ils effectuent ne correspond pas toujours aux normes prescrites par la Haute Autorité de Santé, qui demande au nom de principes éthiques que la femme donne son consentement au test. La plupart des échographistes observés préfèrent suivre les normes professionnelles édictées par le CNTE, qui préconise de faire ce dépistage systématiquement. Leurs réactions à la présentation de nos résultats de recherche ont confirmé notre interprétation. L'un des associés a dit que c'est une «faute professionnelle » de ne pas mesurer la nuque fœtale, en invoquant le rapport du CNTE (Sureau et Henrion, 2005). Les autres échographistes sont restés silencieux sur ce point. Suivre les normes du CNTE leur permet de viser l'excellence professionnelle, en apportant aux autres praticiens qui suivront la grossesse une information précise sur un point jugé essentiel, et de se protéger juridiquement en cas d'éventuels contentieux des parents pour un handicap non décelé. Sur les 28 consultations analysées, 27 comportent un accord pour la réalisation du dépistage, et une seule un accord pour ne pas l'effectuer. Parmi ces 27 consultations, on constate cependant 
qu'il n'y a pas toujours une demande explicite de consentement au test. Dans certains cas, l'opérateur cherche à obtenir rapidement une forme d'accord avec la femme enceinte, en posant une question en rapport avec le dépistage, mais pas sur le test lui-même, par exemple sur la lecture de la fiche d'information ou sa compréhension, ou le fait d'avoir déjà effectué le dépistage pour une grossesse antérieure.

L'absence de demande explicite de consentement dans certaines consultations s'explique en partie par l'aspect contradictoire des normes qui régissent le travail des échographistes. Mais d'autres facteurs peuvent aussi intervenir, comme par exemple le statut exceptionnel de la trisomie 21, devenue emblème du handicap mental dans notre société. Dans toutes les consultations de notre échantillon, l'échographiste mentionne la trisomie 21 sans expliquer en quoi cette anomalie consiste. Il suppose que la femme enceinte la connait, et la femme, ou son compagnon, ne pose jamais de question à ce sujet.

Notre recherche montre également le caractère inadapté des recommandations émises par la Haute Autorité de Santé en ce qui concerne le meilleur moment pour obtenir le consentement au dépistage. Elle préconise de demander l'avis de la femme avant de réaliser le test. Nos observations montrent que les échographistes n'évoquent le sujet à ce moment préliminaire que dans un tiers des consultations, et que celles-ci ne comportent pas toujours une demande de consentement explicite. Dans la majorité des consultations, l'échographiste parle pour la première fois du dépistage au moment où il commence à le réaliser, c'est-à-dire quand il voit le bon profil foetal à l'écran. Dans deux consultations, il aborde même le sujet après avoir effectué la mesure de la nuque fœtale. Mais évoquer le dépistage au moment où on le réalise, ou même après, ne signifie pas pour autant l'imposer à la femme enceinte. Les professionnels qui évoquent le sujet tardivement peuvent demander explicitement le consentement de la femme au test.

Les résultats de notre recherche ne sont pas généralisables car elle n'a été effectuée que dans un centre d'échographies. De plus nous n'avons pas étudié le point de vue des femmes enceintes sur le déroulement de leur consultation, ce qui aurait nécessité un dispositif d'enquête plus contraignant et peut-être irréalisable dans ce cadre. Nous ne savons donc pas si la communication est marquée par des malentendus, tels que ceux analysés par S. Fainzang (2006), mais nos observations laissent penser que l'expression "consentement résigné », qu'elle a forgée pour parler du consentement aux soins dans des services hospitaliers, s'applique à certaines consultations d'échographie prénatale, où la femme enceinte hésitante est persuadée d'accepter. Notre dispositif d'enquête ne nous permet pas de savoir non plus si le fait de donner un consentement au dépistage correspond aux souhaits de toutes les femmes enceintes. Nous ne pouvons pas savoir si les femmes pour qui le dépistage est fait sans qu'elles aient donné un consentement explicite, acceptent cette situation par soulagement de ne pas avoir à prendre la responsabilité morale de consentir au dépistage, par méconnaissance de leurs droits ou pour d'autres raisons encore. Mais même si consentir implicitement correspond aux vœux d'une partie d'entre elles, faudrait-il se satisfaire de cette situation ?

Malgré ces limites, notre recherche montre que l'une des critiques portées au dépistage prénatal de la trisomie 21, à savoir son caractère parfois imposé, est fondée. D'autres recherches sont nécessaires pour savoir si ce phénomène est répandu, en matière d'échographie comme de dépistage par marqueurs sériques, et s'il perdure malgré le changement technique, comme l'introduction du dépistage combiné (échographie et marqueurs) au premier trimestre.

\section{REFERENCES BIBLIOGRAPHIQUES}


ANAES, 1998, L'échographie obstétricale au cours de la grossesse en l'absence de facteur de risque, Rapport de l'Agence Nationale d'Accréditation et d'Evaluation en Santé.

ANAES, 2000, Information des patients. Recommandations destinées aux médecins, Rapport de l'Agence Nationale d'Accréditation et d'Evaluation en Santé.

Champenois-Rousseau B., 2003, Ethique et moralité ordinaire dans la pratique du diagnostic prénatal, Thèse de doctorat de socio-économie de l'innovation, ENSM Paris.

Clarke A., Fishman J., Fosket J., Mamo L., Shim J., 2000, Technosciences et nouvelle biomédicalisation : racines occidentales, rhizomes mondiaux. Sciences sociales et santé, 18, $2,11-42$.

De Vigan C., Khoshnood B., Cadio E., Vodovar V., Goffinet F., 2008, Le registre des malformations de Paris : un outil pour la surveillance des malformations et l'évaluation de leur prise en charge, Bulletin Epidémiologique Hebdomadaire, 28, 250-254.

Ducournau P., 2010, Mettre en banque l'ADN. Enquête sur une biopolitique du consentement, Paris, L'Harmattan.

Fainzang S., 2006, La relation médecins-malades : information et mensonge, Paris, PUF.

Favre R., 2007, En quoi le niveau de connaissance médicale et la position des médecins respectent-ils ou non le consentement des patientes dans le cadre du dépistage de la trisomie 21 ? , Thèse de droit, santé et éthique médicale, Université Paris 5.

Fellous M., 1988, La révolution échographique, Sociologie du travail, n² , 301-322.

Guyard L., 2002, Consultation gynécologique et gestion de l'intime, Champ psychosomatique, 27, 81-89.

HAS, 2005, Comment mieux informer les femmes enceintes. Recommandations pour la pratique clinique, Rapport de la Haute autorité de santé.

HAS, 2007, L'évaluation des stratégies de dépistage de la trisomie 21, Rapport de la Haute autorité de santé.

Heyman B., Hundt G., Sandall J., Spencer K., Williams C., Grellier R., Pitson L., 2006, On being at higher risk: A qualitative study of prenatal screening for chromosomal abnomalies, Social Science and Medicine, 62, 10, 2360-2372.

Katz Rothman B., 1989, The tentative pregnancy: how amniocentesis changes the experience of motherhood, New York, Norton.

Khoshnood B., De Vigan C., Blondel B., Lhomme A., Vodovar V., Garel M., Goffinet F., 2006, Women's interpretation of an abnormal result on measurement of fetal nuchal translucency and maternal serum screening for prenatal testing of Down syndrome, Ultrasound in Obstetrics and Gynecology, 28, 3, 242-248.

Leblanc P., 2010, Peut-on encore refuser le dépistage de la trisomie 21 ? Abstract Gynécologie, 341, 12-14.

Marteau T.M., Slack J., Kidd J., Shaw R.W., 1992, Presenting a routine screening test in antenatal care : practice observed, Public Health, 106, 2, 131-41.

Marzano M., 2006, Je consens, donc je suis ... . Ethique de l'autonomie, Paris, PUF.

Mattei Jean-François, 2000, Le diagnostic prénatal, Paris, Flammarion.

Memmi D. 2003, Faire vivre et laisser mourir. Le gouvernement contemporain de la naissance et de la mort, Paris, La Découverte.

Ménoret M., 2007, Informer mais convaincre : incertitude médicale et rhétorique statistique en cancérologie, Sciences Sociales et Santé, 25, 33-52.

Milliez J., 1999, L'euthanasie du foetus, médecine ou eugénisme?, Paris, Odile Jacob.

Moyse, D., 1999, Le risque de naître différent, Esprit 12, 65-74.

Peretz H, 1998, Les méthodes en sociologie : l'observation, Paris, La Découverte.

Pilnick A., 2008, It's something for you both to think about': choice and decision making in nuchal translucency screening for Down's syndrome, Sociology of Health and Illness, 30, 4, 511-530. 
Pinell P., 2004, Dépistage, In : Lecourt D., Dictionnaire de la pensée médicale, Paris, PUF, 320-24.

Press N., Browner C., 1997, Why women say yes to prenatal diagnosis, Social Science and Medicine, 45, 7, 979-989.

Rapp R., 2000, Testing women, testing the fetus. The social impact of amniocentesis in America. New York, Routledge.

Seguy B., 2006, Prévenir le risque juridique en obstétrique. Cas réels, bonnes pratiques, Paris, Masson.

Seror V., Ville Y., 2009, Prenatal screening for Down syndrome: women's involvement in decision-making and their attitudes to screening, Prenatal Diagnosis, 29, 120-128.

Sicard D., 2007, La France au risque de l'eugénisme, Le Monde, 5 février.

Sureau C., Henrion R., 2005, Rapport du Comité national technique de l'échographie de dépistage prénatal.

Strauss A., 1992, La trame de la négociation. Sociologie qualitative et interactionnisme, Textes réunis et présentés par I. Baszanger, Paris, L'Harmattan.

Vassy C., 2011, De l'innovation biomédicale à la pratique de masse : le dépistage prénatal de la trisomie 21 en Angleterre et en France, Sciences sociales et santé, 29, 3, 5-32.

Williams C., Alderson P, Farsides B., 2002, Is non-directiveness possible within the context of antenatal screening and testing? Social Science and Medicine, 54, 3, 339-47.

\section{Remerciements :}

Nous remercions les femmes enceintes et les praticiens qui ont accepté que nous les observions lors des consultations, ainsi que l'Agence de la Biomédecine qui a financé ce projet de recherche (2008-20010) mené sous la responsabilité scientifique de Carine Vassy.

\section{Summary:}

Pregnant women in France are routinely offered Down's syndrome screening tests. These tests and further testing to confirm the diagnostic, may lead to the decision to terminate the pregnancy. Fetal ultrasound is one of the most frequently used techniques to screen pregnancies. Public authorities have set guidelines for practitioners to ask pregnant women if they consent to Down's syndrome screening before having the test. However, some critics argue that pregnant women have actually no choice anymore but to undergo the test. We have conducted a sociological fieldwork in an ultrasound scanning clinic in Paris area and analyzed what sonographers say to pregnant women. Getting implicit consent from pregnant women is good enough for most sonographers.

Key-words: fetal ultrasound scan, screening, consent, Down's syndrome, ethics 
Tableau : Moment de première évocation du dépistage de la trisomie 21 dans la consultation échographique

\begin{tabular}{|c|c|c|c|}
\hline $\begin{array}{c}\text { Moment de première } \\
\text { évocation du dépistage }\end{array}$ & $\begin{array}{l}\text { Nombre } \\
\text { de } \\
\text { consultations }\end{array}$ & $\begin{array}{c}\text { Nom fictif des } \\
\text { opérateurs }\end{array}$ & $\begin{array}{l}\text { Nombre } \\
\text { d'opérateurs } \\
\text { concernés }\end{array}$ \\
\hline Introduction & 10 & B, C, F & 3 \\
\hline $\begin{array}{c}\text { Examen } \\
\text { échographique }\end{array}$ & 16 & A, C, D, E, G & 5 \\
\hline Conclusion & 2 & C, I & 2 \\
\hline Total & 28 & - & - \\
\hline
\end{tabular}

